\title{
The Theory of Kindergarten
}

\author{
Wujin Gaowa',a , Tuoya ${ }^{2, b}$, Wenhui Ming ${ }^{3, b}$ \\ ${ }^{1}$ Inner Mongolia University of Science and Technology, Baotou, Inner Mongolia 014010, China \\ ${ }^{2}$ Baotou City Chengxinda Engineering Consulting \& Supervision co., LTD, Baotou, Inner \\ Mongolia 014010, China \\ ${ }^{3}$ Baotou City Planning Bureau, Baotou, Inner Mongolia 014010, China \\ a2230638411@qq.com_b826317991@qq.com
}

Keywords: kindergarten, master plan design, functional division, play area outside.

Abstract: Kindergarten should create graceful environment to fit in children's psychological request. In designing master plan of kindergarten, a favorable exterior environment should be created by functional division, entrance setting, building organization and integration of exterior play area, green and roads.Kindergarten is not only a heaven of children, but also a place where they grow up and learn knowledge.Kindergarten design should confirm to the rule of education, the rules of children's growing and the feature of environment they live in. In master plan design everything should start with fitting in children's age, feature and genders, so that the environment can give a full play to both the influence and the education they get.

\section{Site selection}

The condition of kindergarten site is the prerequisite of its exterior environment.

Kindergarten should have an independent site, in site selection local climate and environment surroundings should take into account.

Site selection of kindergarten should meet requirements as follows,

1) Keep away from all kinds of pollution, meet the request of health protection standard.

2) An appropriate distance for parents convenience, avoid traffic jam.

3) Abundant sunshine, dry site, clear drainage, favorable environment or next to city green land.

4) Supply basic condition to the arrangement of functional division, entrance and playground outside.

\section{Master plan organization}

The design of kindergarten master plan includes: functional division, entrance setting, building arrangement, play area outside design, sundries storage and organization of both green and road.

Functional division

According to its usage requests, land for kindergarten includes: construction land, playing area, land for road, green land and sundries storage yard, etc. Construction land includes land for living room, service room and supply. Playing area includes playground, wading pool, sand pool, plantation and room for little animals.

The properties of each function should be clearly divided and avoid confliction, however, the close parts in function should be connected, and pay attention to making full use of transition space. 


\section{Entrance setting}

Normally there are two entrances in medium- and large-scale kindergartens. The main entrance sever for parents and children while the secondary entrance for sundries storage, the position for entrance should be determined by terrain and roads, which shouldn't be set close to road intersection. The width of the entrance can't be less than $4 \mathrm{~m}$ (see Fig.1).

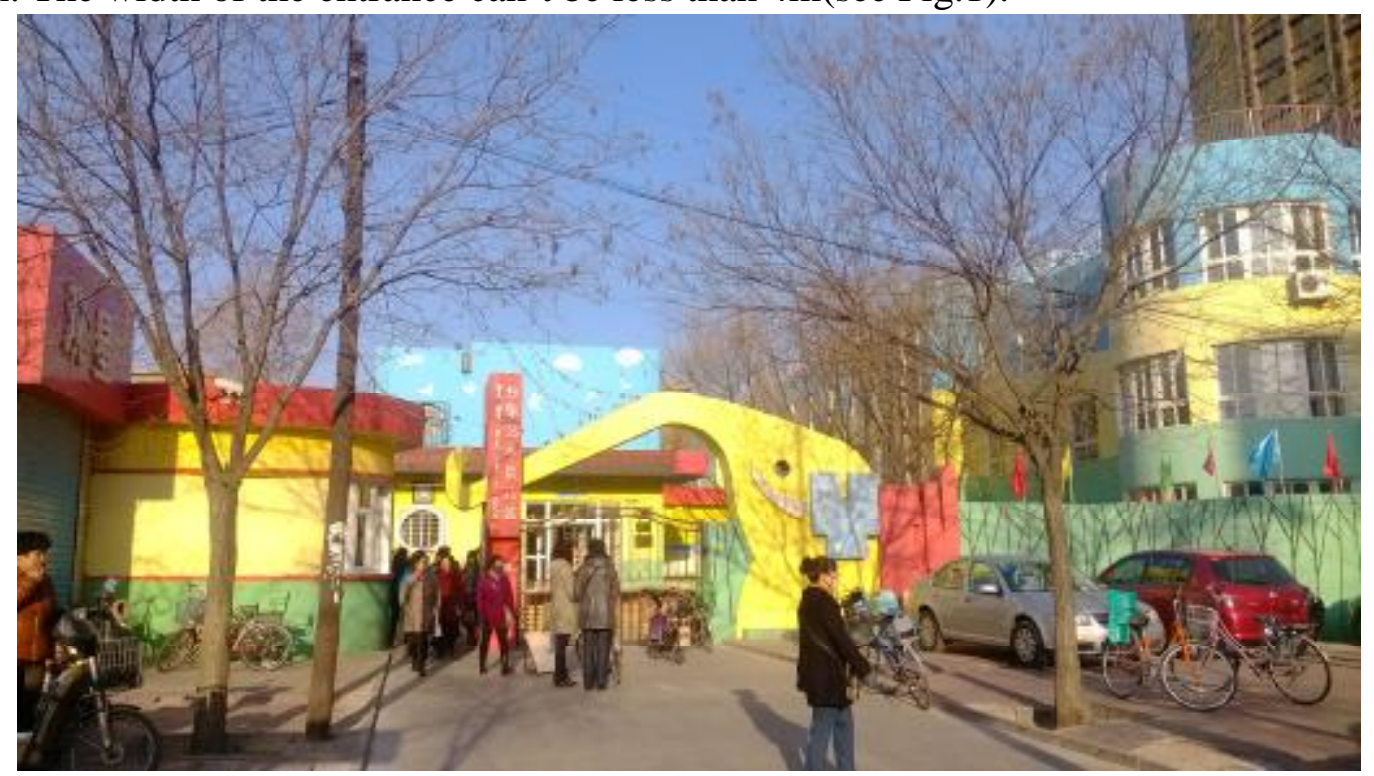

Fig.1 the main entrance

The design of the entrance should fully embody the features of kindergarten buildings. To create intimacy of its accessible, the size of the entrance should be light, and it's better if it could be permeable, what's more, iconic decoration is necessary when combine it to children's interests. To avoid children's climbing and getting through; gates and enclosure should make use of vertical fencing. The one concave is more acceptable and attractive than the one bulged out. The width of the entrance should meet the request of its circulation, transportation and fire protection. Normally it is not less than $4 \mathrm{~m}$; the main entrance of site should bear a direct relationship to the entrance of buildings in the site, and it is of great importance that pays more attention to separate the pedestrian flow and traffic flow, avoid interruption and roundabout ways; in addition, the safety design should be paid most attention.

\section{Building arrangement}

The plan of kindergarten should be functional divided clear, avoid interruption, facilitate both use and management, and be good for traffic evacuation. The designer should pay special attention to applying a the best position of solar condition for living room, what's more, the living room should meet the requirement that lowest window can get at least 3 hours sunshine in winter solstices(see Fig.2). 

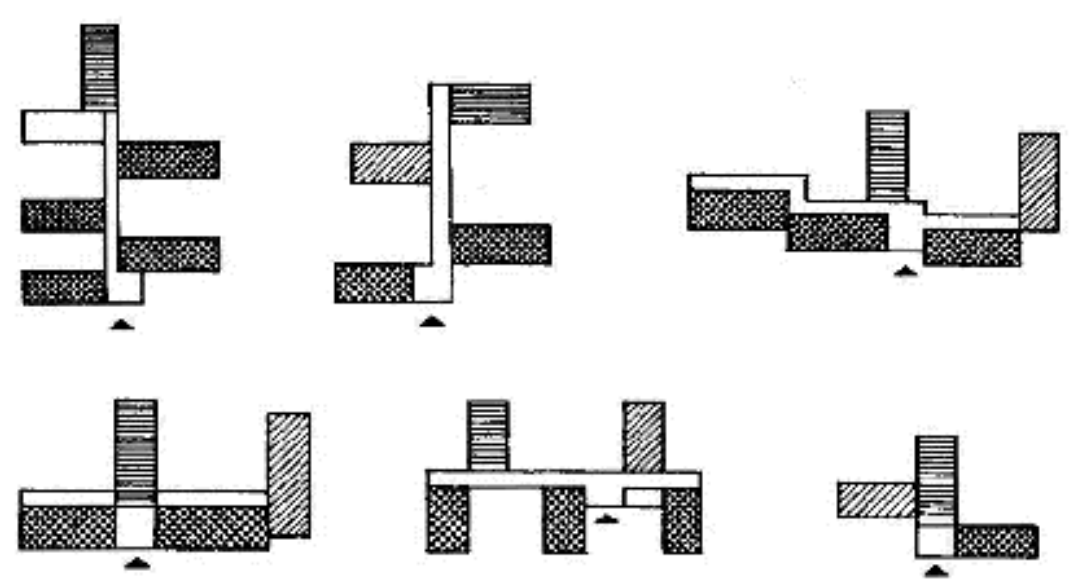

Fig.2 kindergarten plan

Playground outside design

Fencing

The fencing for separating kindergarten from outside has effect to protected the kindergarten, and the one of permeable is the best choice, bring the landscape outside to children's view while stay inside, which is both the connection and the separation. It facilitate the children enjoy landscape outside, not only satisfy their curiosity but also create the intimacy of neighborhood. The parapets and plants can also be used in space division, by combining them with game facilities in design, variety space could be create.

Game and play area design

The most important way for children's grow-up is group game, they gain knowledge in playing game. However playing outside is more important than many activities inside.

In designing kindergarten plan it is necessary that setting dedicated play areas outside for every class. The playground for each class should be more than $60 \mathrm{~m}^{2}$, usually it is hard ground. The different playing areas should be separated, meanwhile there should be a few public play areas for children in kindergarten, the public play area outside may set game facilities like 30-meter track, sand pit, washing basin and wading pool of no more than $0.3 \mathrm{~m}$ depth, etc. In addition the site should be set in the position where few pedestrian flows.

In designing play area of kindergarten, analysis for children's psychology and action should be taken into consideration, which includes content of game, game routine and the influence the type of game facility bring to children. What's more, the change in the size and the height, the plants and the logo marking should meet the requirement of children's psychology.

The geography and the pavement

The variety height of geography is an important element for play area. In landscape planning, it is necessary to provide children independent space of voice and version in nature area, playing area and the gap between facilities, add suitable landscape in playing area.

Pavement of ground should take children's safety into consideration, pay attention to the durability, harmlessness and non-skid property of the materials, what's more, the material should be hypoallergenic, and can be used in different weather. And of course, they should look good.

Green and road arrangement

Children could be inspired to participate in lots of creative activities by planting trees and 
flowers, setting paths and hills as well as building fencing and water pools. Keep all the natural landscape, includes trees, water pool and natural slope, etc. Make full use of different size and shape of the green of kindergarten.

The arrangement of the road in kindergarten should avoid the interruption from the traffic flow.

\section{Conclusion}

Master plan design of kindergarten should follow the principle of adjusting measures to local conditions. Avoid blind angle and make full use of site by carefully design and planning.

The key points of master plan designing are making clear of program, convenient transportation, large amount of green and rational fire proof design, etc. it can make children enjoy sunshine and close to nature while playing outside.

\section{References}

[1] Architectural design information set 3. (Second edition). China Building Industry Press.

[2] Huang Jun-rong,On Building Design for Modern Kindergarten,Guangxi Town Construction, 2009 (6)

[3]Zhao Chen-mei,Application of architectural behavioristic psychology in the design of kindergarten building, Shanxi architecture, May.2012 